\title{
The face of appearance-related social pressure: gender, age and body mass variations in peer and parental pressure during adolescence
}

\author{
Susanne Helfert ${ }^{*}$ and Petra Warschburger
}

\begin{abstract}
Background: Appearance-related social pressure plays an important role in the development of a negative body image and self-esteem as well as severe mental disorders during adolescence (e.g. eating disorders, depression). Identifying who is particularly affected by social pressure can improve targeted prevention and intervention, but findings have either been lacking or controversial. Thus the aim of this study is to provide a detailed picture of gender, weight, and age-related variations in the perception of appearance-related social pressure by peers and parents.

Methods: 1112 German students between grades 7 and 9 (mean age: $M=13.38, S D=.81$ ) filled in the Appearance-Related Social Pressure Questionnaire (German: FASD), which considers different sources (peers, parents) as well as various kinds of social pressure (e.g. teasing, modeling, encouragement).

Results: Girls were more affected by peer pressure, while gender differences in parental pressure seemed negligible. Main effects of grade-level suggested a particular increase in indirect peer pressure (e.g. appearancerelated school and class norms) from early to middle adolescence. Boys and girls with higher BMI were particularly affected by peer teasing and exclusion as well as by parental encouragement to control weight and shape.

Conclusion: The results suggest that preventive efforts targeting body concerns and disordered eating should bring up the topic of appearance pressure in a school-based context and should strengthen those adolescents who are particularly at risk - in our study, girls and adolescents with higher weight status. Early adolescence and school transition appear to be crucial periods for these efforts. Moreover, the comprehensive assessment of appearancerelated social pressure appears to be a fruitful way to further explore social risk-factors in the development of a negative body image.
\end{abstract}

Keywords: Peer pressure, Parental pressure, Adolescence, Gender, Age, BMI

Factors influencing the development of a negative body image during adolescence have received increasing attention due to the fact that body dissatisfaction is highly prevalent among adolescents in western society and is also one of the main predictors of low self-esteem, depression, and not least of all disordered eating [1-3]. Sociocultural influences are particularly relevant in this process. Thompson's Tripartite Influence Model [4] of body dissatisfaction and Stice's Sociocultural Model of Disordered Eating [5] have identified media, peers, and

\footnotetext{
* Correspondence: srudolf@uni-potsdam.de

Department of Psychology, University of Potsdam, Karl-Liebknecht-Str. 24/25, 14476, Potsdam, OT Golm, Germany
}

\section{() BioMed Central}

(c) 2013 Helfert and Warschburger; licensee BioMed Central Ltd. This is an Open Access article distributed under the terms of the Creative Commons Attribution License (http://creativecommons.org/licenses/by/2.0), which permits unrestricted use, distribution, and reproduction in any medium, provided the original work is properly cited. parents as the three formative sociocultural influences. Many studies have emphasized the crucial role of perceived appearance-related social pressure in the development of body dissatisfaction and disordered eating. Thus, social agents - especially peers and parents, who are closest to the adolescent - both consciously and unconsciously convey and enhance appearance-related norms through direct and indirect interactions [5,6]. Peers and parents promote the construction of beauty ideals, norms, and standards and highlight the importance of appearance. Numerous studies have investigated different aspects of peer [e.g. 1,7-9] and parental pressure [e.g. 10-16]. However, to our knowledge no theoretical framework has 
yet integrated the main influences from both peers and parents discussed in the literature. In order to develop a comprehensive measure of appearance-related pressure from peers and parents (see [17]), we reviewed the literature and found influences from friends [1,2] and schoolmates as well as teasing or exclusion to be the most established peer influences. With regard to parental influences, aspects such as parental norms and modeling behavior regarding appearance [e.g.10-12], parental disregard or ignorance [e.g.13] as well as teasing [e.g.9,14] and encouragement to control weight and shape [e.g.13,15,16] have been found to affect the body image of adolescents (see Figure 1).

Up to now, research has provided important findings on the impact of single types of social pressure and general behavioral mechanisms. However, in order to explain the development of negative body image and design targeted prevention approaches, we must also find out who is particularly faced with social pressure. The following sections will attempt to summarize the knowledge on variations according to individual characteristics considering gender-, age, and weight-related variations.

\section{Gender variations}

Because studies on social pressure have mostly derived from eating disorder and body image research, they have often concentrated on girls, for whom they reported a higher amount of appearance-related influences from friends [e.g. 16,18], more fear of exclusion by peers because of one's appearance [19] and a greater importance of school and class norms [20]. These findings appear quite plausible with regard to the particular emphasis placed on female beauty and appearance in western society. However, during the last ten years research has also considered boys and revealed that some of the gender differences might be due to inadequate instruments for boys (i.e., only focusing on the thin ideal $[21,22])$. Consequently, studies that used measures without that bias suggested comparable processes of appearance-related interactions with friends and social exclusion for both girls and boys [7,23].

Findings regarding gender differences involving parental pressure have been sparser but therefore less controversial. They predominantly support the conclusion that appearance is more heavily emphasized among girls. Consequently, girls perceived a greater extent of parental appearance norms and modeling behavior (e.g., parental concerns with body shape, efforts to look good $[6,16,24])$. Interestingly, studies investigating parental encouragement to control weight and shape found no gender difference $[13,16,25]$. However, this might be due to the focus on encouragement to diet, which might be used by parents regardless of their child's gender when the child is at risk of becoming overweight. We suppose that if an operationalization of "encouragement" without the bias towards the thin ideal is applied, gender differences might occur. Concerning parental disregard (i.e., injustice and ignorance) studies are rare. The study of Meesters et al. [13] among Dutch adolescents aged 10 to 16 provided important suggestions regarding the influential role of parental rejection or insecure attachment in the development of body concerns but could not find gender variations. However, this aspect of parental pressure requires further investigation.

Findings on peer and parental teasing have been particularly inconsistent. While in some studies [26] girls were more frequently faced with peer teasing, others did not find any gender difference $[18,27]$ or even found more teasing experiences among boys $[7,16]$. The same applies with parental teasing. Some studies did not find

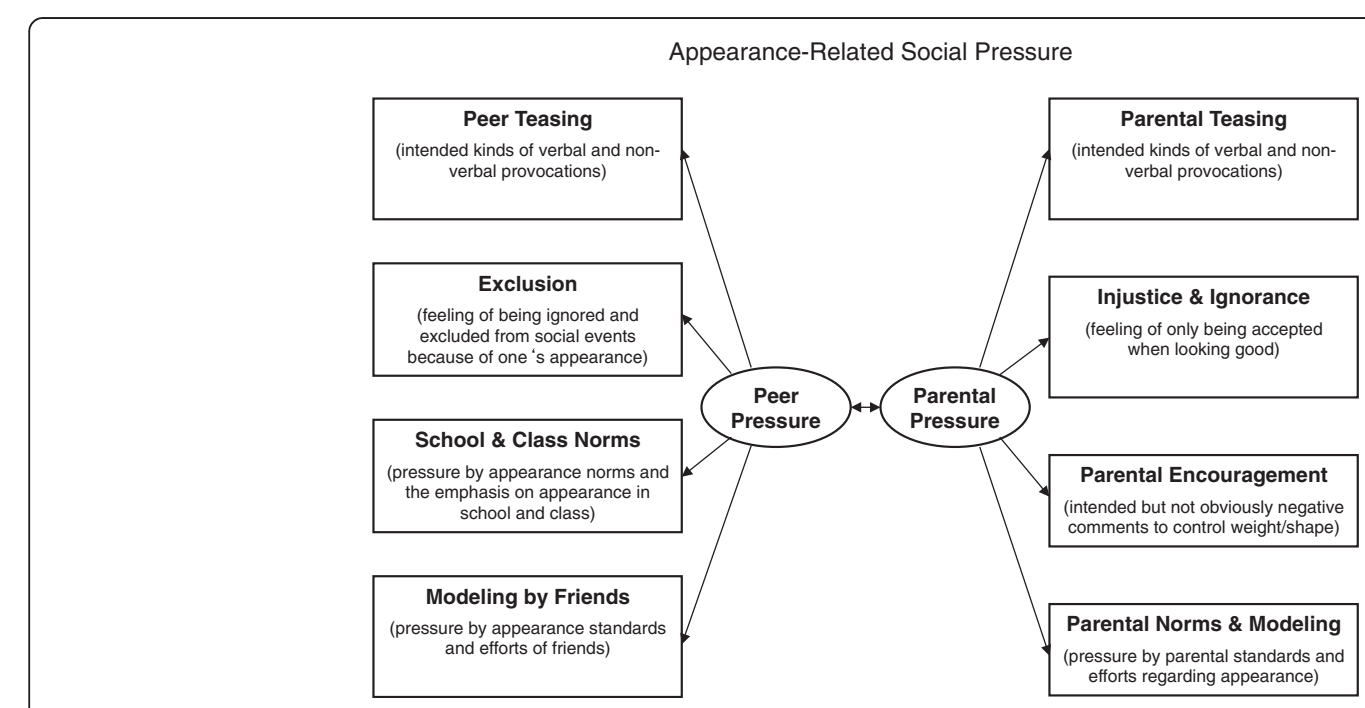

Figure 1 Considered aspects of appearance-related social pressure. 
a gender difference $[6,16]$ and others have revealed that girls perceive more parental teasing [24,27]. These inconsistencies might result from the measurement of teasing as isolated indices or as combinations of peer and parental teasing. Hence, validity and reliability might have been restricted.

\section{Age-related variations}

Developmental theories on the transformation of relationships with peers and parents [28] suggest that social pressure might change throughout adolescence. Further, the gender intensification hypothesis of Hill and Lynch [29] suggests that pressure from peers and parents to conform to gender roles, behavior and appearance standards intensifies during adolescence. However, only a few studies have investigated developmental effects in the field of social pressure and reported a growing influence of friends and an increase in appearance pressure by other peers (e.g., schoolmates) during middle adolescence $[1,7]$. In addition, Dohnt and Tiggemann [30] provided interesting findings on the impact of school and class norms among elementary school girls in the first four years of formal schooling. While girls in the first year at school thought that their peers would desire a larger figure, girls from grade two to four already assumed that their peers desired a thinner figure. These results suggest that orientation towards a certain body ideal as well as appearance-related school and class norms develop very early. Interestingly, Chen and Jackson [31] reported an age-gender interaction among a sample of Chinese adolescents, suggesting that appearance conversations between friends might increase with age only among girls but not among boys. However, they could not establish a comparable effect regarding general appearance-related pressure. In contrast to a probable increase in appearance-related interactions, teasing and exclusion proved to be rather stable during adolescence [7]. Jones [1] even found a decrease in reported teasing among adolescents from grades 10 to 11, which indicates that teasing becomes less important with the transition to adulthood.

To our knowledge, no study exists that considered age-related variations in parental pressure, but developmental theories have suggested a decrease in adult orientation and an increase in peer orientation for appearance-related issues beginning in early adolescence $[28,32,33]$. This might lead to the conclusion that parental pressure has either a stable or even a shrinking relevance during adolescence. However, Striegel-Moore and Kearney-Cooke [34] revealed that American parents become more critical of their children's physical attractiveness as the children grow older. Hence, appearancerelated pressure (e.g., encouragement to control weight and shape) might also increase.
However, because findings on parental pressure have been incomplete and knowledge of age-related trends in peer pressure comes from a few predominantly crosssectional studies, we should be cautious about drawing conclusions for age-related trends.

\section{Body mass variations}

Many studies have examined stigmatization of overweight and obese persons. As appearance stigmatization is a distinct and serious form of social pressure, including peer teasing and exclusion alike, it can be concluded that overweight persons per se experience more of these kinds of pressure [9]. Beyond that, a few studies have also suggested a higher amount of teasing experiences among underweight adolescents [26]. The results of Jones and Crawford [7] even suggest an interaction of weight and gender: While particularly overweight girls experienced teasing and fear of exclusion, underweight boys displayed the highest scores. These results were interpreted with regard to the different beauty ideals for men and women: Girls who do not fit the slim norm and boys who do not fit the bulky, muscular male ideal are more exposed to stigmatization. However, the findings have left the question unanswered whether deviating from normal weight per se increases the risk of being subjected to more direct peer pressure or whether weight-related variations are different for girls and boys.

To our knowledge, only Jones and Crawford [7] have considered weight variations in more subtle forms of peer pressure and found that adolescents with higher BMI perceived stronger influences from friends and general appearance pressure by peers (e.g., schoolmates).

Studies reporting relationships between weight status and parental pressure are even sparser. A few studies reported higher scores in parental teasing among overweight boys and girls [24,26,35]. Regarding parental encouragement to lose weight, Wertheim et al. [25] found a moderate positive association with weight status for early adolescent boys and girls alike. Unfortunately, the study did not consider muscle gaining. Finally, Rodgers et al. [24] could not find an association between weight status and the perception of appearance-related parental norms and modeling behavior.

In summary, more knowledge on variation according to individual characteristics is needed to explain the development of negative body image and to design targeted prevention approaches. While previous studies have provided important findings on the impact of single types of social pressure and general behavioral mechanisms, findings on gender, age and weight variations in different aspects of social pressure have either been incomplete or controversial, because only a few studies have explicitly focused on these individual differences. Moreover, due to restricted sample size most of the studies could not 
consider possible interactions between the three factors. Finally, research has often concentrated on girls, or when it included boys, the applied measures often contained a bias towards the thin ideal that is not suitable for boys.

Thus, research still remains limited for the purpose of drawing firm conclusions about gender, body mass variations and age-related trends in the perception of social pressure.

\section{Hypotheses}

The current study attempts to contribute to an enhancement of current theories on appearance-related social pressure by investigating the occurrence of different types of pressure in a large sample of German adolescent boys and girls. Moreover it provides a comprehensive exploration of differential effects of gender, weight, and grade as well as interactions among these factors. Based on previous findings, we expected the following:

\section{Gender variations}

1. The research of the recent years has posed the question whether the emphasis placed on female beauty sets girls at greater risk for appearancerelated social pressure or whether these effects have derived from biased instruments that were unsuitable for boys. Even if several studies have pointed to the growing relevance of appearance among boys and some gender differences diminished when studies use muscle- and weight-related instruments, most of the findings suggest that the focus on appearance is still stronger for females. Consequently, we hypothesized that girls would show higher levels of peer pressure through modeling by friends, school and class norms, peer teasing and exclusion as well as higher levels of parental pressure through parental teasing, encouragement to control weight and shape, parental norms and modeling and injustice and ignorance.

\section{Grade-level variations}

2. Previous findings have brought evidence for an agerelated increase of appearance orientation and modeling processes among adolescents whereas more direct aspects of peer pressure have proven to be quite stable. We thus hypothesized that modeling by friends and perceived school and class norms would be higher in older compared to younger adolescents. To take account of the findings of Chen and Jackson [31] we also want to test for an interaction between age and gender.

3. Regarding parental pressure, findings are rare and therefore we based our expectations on developmental theories. These theories have suggested that parents are not the main source of appearance-related standards and thus parental norms and modeling should not differ by grade. However, parents have been found to become more concerned with the physical attractiveness of their adolescent child. Thus, we expected that parental encouragement to control weight and shape would be more prevalent among older adolescents.

\section{Body mass variations}

4. Finally, research has raised the hypothesis that either a) higher weight status per se sets individuals at greater risk for stigmatization or b) girls with higher weight are stigmatized if they do not fit the female slim ideal, whereas boys experience teasing and exclusion if they are too thin and do not fit the male muscular ideal. As the majority of studies have found evidence for the first hypothesis, we predicted that overweight girls and boys would report higher levels of all kinds of peer pressure (i.e. teasing, exclusion, influences by friends, pressure from school and class norms).

5. Based on previous studies we further expected that overweight adolescents would experience more parental teasing as well as encouragement to control weight and shape.

\section{Method}

\section{Participants and procedure}

This study reports on the baseline survey of a longitudinal investigation for which the procedure was approved by the ethics commission and the local ministry of education. The study was conducted among middle- and upper-class students in grades 7 to 9 in six German high schools that cooperate with our institution for different research projects. Teachers delivered written information to the students and their parents and collected informed consent forms from those who agreed to participate. Of the 1,342 students who received information, 1,113 (83\%) returned their consent forms and completed the questionnaire during a regular lesson. One case was excluded due to invalid data. Demographic information for the remaining sample of 1,112 students is given in Table 1 .

\section{Measures}

\section{Weight status}

Body-mass index (BMI) was computed based on selfreported age, weight, and height. Self-reported weight is proven to be a valid measure in epidemiological studies with adolescents [36]. The percentile ranking of BMI was assigned using the WHO norms for age and gender [37]. Following Jones and Crawford [7] weight status 
Table 1 Demographic Characteristics of the Sample

$(\mathrm{N}=1,112)$

\begin{tabular}{|c|c|c|c|c|c|}
\hline \multirow{3}{*}{ Age } & \multicolumn{2}{|c|}{ Girls $(n=603)$} & \multicolumn{2}{|c|}{ Boys $(n=509)$} & $p$ \\
\hline & \multicolumn{2}{|c|}{$10-16$ years } & \multicolumn{2}{|c|}{$11-16$ year } & .003 \\
\hline & \multicolumn{2}{|c|}{$M=13.32, S D=0.79$} & \multicolumn{2}{|c|}{$M=13.46, S D=0.83$} & \\
\hline \multirow[t]{3}{*}{ Grade } & $36.7 \%$ & grade 7 & $33.8 \%$ & grade 7 & n.s. \\
\hline & $36.3 \%$ & grade 8 & $36.9 \%$ & grade 8 & \\
\hline & $27.0 \%$ & grade 9 & $29.3 \%$ & grade 9 & \\
\hline BMI & \multicolumn{2}{|c|}{$M=18.63, S D=2.57$} & \multicolumn{2}{|c|}{$M=18.70, S D=2.73$} & n.s. \\
\hline
\end{tabular}

was classified as follows: underweight $\left(\mathrm{BMI}<25^{\text {th }}\right.$ percentile), low average weight $\left(25^{\text {th }} \leq \mathrm{BMI}<50^{\text {th }}\right.$ percentile), high average weight $\left(50^{\text {th }} \leq \mathrm{BMI}<85^{\text {th }}\right.$ percentile $)$, and overweight (BMI $\geq 85^{\text {th }}$ percentile).

\section{Appearance-related social pressure}

The assessment of social pressure has been limited in previous research. Studies that explored mechanisms of sociocultural pressure predominantly asked about a general feeling of pressure to be thin often with single items (e.g. 11,14], The Perceived Sociocultural Pressure Scale [38]). Moreover, several studies applied measures to focus on specific aspects of pressure (e.g., peer influence: Inventory of Peer Influence on Eating Concerns (IPIEC [19]); family influence: Family Influence Scale (FIS [39]); and teasing: Perception of Teasing Scale (POTS [40]). Because most of these items imply a connotation towards a thin ideal, they are probably not suitable among boys and might thus lead to underestimations of the relevance of pressure among boys.

Because to our knowledge no instrument exists that measures social pressure from peers and parents simultaneously while distinguishing various types of pressure, we developed the Appearance-Related Social Pressure Questionnaire (FASD, Fragebogen zum aussehensbezogenen sozialen Druck [17]). To gather an accurate measure of social pressure we included on the one hand those social impacts established in the literature and on the other hand conducted qualitative interviews with adolescent girls and boys exploring important sources of social pressure in their daily lives. The literature predominantly provides evidence for comparable risk factors for body concerns in both boys and girls [e.g. 41,42]. The findings from our interviews during the item generation also pointed to comparable forms of social pressure. However, we had to ensure that the phrases were suitable for both girls and boys as well as for adolescents with different weight statuses. Thus, we used general terms like "appearance" or "body shape" and tried to avoid specific ones like "thinness" to avoid a bias. The 32 items are rated on a 5-point Likert scale from 1 (strongly disagree) to 5 (strongly agree). A series of structural equation models was used to investigate the factor structure of the FASD. The best fitting model revealed two parts (peer and parental pressure), each consisting of four scales that comprise four items, respectively, and ask about different types of appearance-related social pressure.

The section on parental pressure comprises four scales:

Parental Teasing $\left(\alpha=.83, r_{t t}=.60\right)$ : This scale combines direct aspects of pressure from parents such as negative comments or disparaging gestures.

Injustice and Ignorance $\left(\alpha=.65, r_{t t}=.72\right)$ : By measuring the feeling of only being accepted when looking better or being ignored for not looking good, the second scale implies an indirect kind of pressure. Although we could not find previous literature that directly investigated this parental impact, it was mentioned by the adolescents that were interviewed during the construction of the FASD, and the findings of Meesters et al. [13]. also suggested such aspects of parental pressure.

Parental Encouragement to Control Weight and Shape $\left(\alpha=.79, r_{t t}=.81\right)$ : The third scale includes also direct - but in contrast to the first scale, not obviously disparaging - comments by parents as it measures parental encouragement to pay heed to one's body shape.

Parental Norms and Modeling $\left(\alpha=.74, r_{t t}=.83\right)$ : Finally, the fourth parental scale comprises indirect pressure through parental standards of appearance and efforts to look good.

The section peer pressure comprises the following four scales:

Peer Teasing $\left(\alpha=.78, r_{t t}=.83\right)$ : Comparable to the parental scale, this scale is composed of direct types of pressure like disparaging comments and gestures by peers.

Exclusion $\left(\alpha=.81, r_{t t}=.86\right)$ : This scale asks about the feeling of being ignored or excluded from social events because of one's appearance.

School and Class Norms $\left(\alpha=.78, r_{t t}=.69\right)$ : The third scale measures an indirect aspect of peer pressure as it inquires about the importance of appearance in school and class.

Modeling by Friends $\left(\alpha=.73, r_{t t}=.72\right)$ : The final peer pressure scale asks about appearance standards of friends and efforts to achieve that standard, which can also be seen as an indirect aspect of peer pressure.

The internal consistency scores were taken from the current sample, whereas the test-retest reliability coefficients were obtained in a previous study. Intercorrelations between the FASD-scales in this study were predominantly moderate ( $r=.13$ to .55 ). Only teasing by peers and exclusion showed a higher association $(r=.68)$. The FASD 
has been used in different studies to ensure its psychometric quality [e.g. 17,43]. Reliability was acceptable for all scales and evidence for factorial, convergent, and incremental validity has been determined [17]. Details on the construction and validation of the FASD are available on:

http://www.psych.uni-potsdam.de/counseling/research/messure-e.html.

\section{Statistical analyses}

All statistical analyses were performed using SPSS 15.0. Because missing data rates were below 5\% common EMsubstitution was applied. We conducted preliminary analyses using ANOVA and the chi square test to investigate the characteristics of the sample and differences in the group formation. In order to investigate differential effects in the perception of different types of social pressure we conducted a 2 (gender) x 3 (grade-level) x 4 (BMI category) multivariate analysis of variance (MANOVA) including the mean scores of all FASD subscales. We decided to include gender, grade-level, and BMI in one analysis, because different authors have discussed interactive effects of gender, weight, and age and, moreover, we wanted to account for confounding effects because our data suggested associations between the factors. Furthermore, MANOVA was chosen due to the substantial intercorrelations among the different FASD scales. Wilks' Lambda will be reported as the multivariate test criterion. For the post hoc univariate analysis, the significance level was adjusted using Bonferroni correction $(p<.006)$

\section{Results}

\section{Preliminary analyses}

Preliminary analyses revealed that the boys in our sample were slightly older, $t(1110)=2,94, p<.01$, and significantly more of them could be classified as being overweight, $x^{2}$ $(3, \mathrm{n}=1112)=9.17, p<.05$ (Table 2). In addition, students in grades 7 to 9 significantly differed as to mean age with only marginal overlaps in range, $F(2,1109)=1237.50$, $p<.001$. Hence, the mean age in grade 7 was: $M=12.66$ $(S D=0.43)$, in grade $8: M=13.33(S D=0.45)$ and in grade 9: $M=14.36(S D=0.47)$. Finally, analyses indicated that BMI significantly increased with age, $F(2,1109)=3.57$, $p<.001$. However, no differences could be found regarding the distribution of the four weight status groups according to gender and grade. With the use of MANOVA, we could account for the variations between the groups.

\section{Overall results}

The overall 2 (gender) x 3 (grade-level) x 4 (BMI category) MANOVA of the different aspects of social pressure did not show a significant overall interaction between gender, grade, and weight status but did reveal main effects for all the three factors. Hence, the MANOVA revealed a significant main effect for gender, $F(8,1081)=16.64, p<.001$, $\eta^{2}=.11$, which was of medium size (Table 3). Furthermore, we found a moderate main effect for grade-level, $F(16,2164)=5.91, p<.001, \eta^{2}=.04$ (Table 4) and a moderate main effect for BMI category, $F(24,3249)=7.01$, $p<.001, \eta^{2}=.05$ (Table 5).

\section{Main effects for gender Hypothesis 1}

With regard to gender effects we expected a main effect indicating that girls display higher levels on all aspects of appearance-related social pressure from peers and parents. However, follow-up univariate tests confirmed the main effect for gender only for one aspect of parental pressure. Hence, girls reported more parental teasing, $F(1,1088)=$ 10.81, $p<.01, \eta^{2}=.01$, which constitutes a small effect. Furthermore, girls displayed higher scores on all peer pressure scales. More specifically, we found small effects regarding peer teasing, $F(1,1088)=13.11, p<.001, \eta^{2}=.01$; exclusion, $F(1,1088)=53.81, p<.001, \eta^{2}=.05$; and school and class norms, $F(1,1088)=29.77, p<.001, \eta^{2}=.03$ but for modeling by friends the effect is even of medium size, $F(1,1088)=72.58, p<.001, \eta^{2}=.06$.

In sum, gender differences in peer pressure were noteworthy and indicated that girls perceived more pressure from peers compared to boys, while the largest difference was revealed for modeling by friends.

\section{Main effects for grade-level \\ Hypothesis 2}

We hypothesized that the impact of friends and schoolmates would be higher in older compared to younger

Table 2 BMI Groups by Gender and Grade $(\mathrm{N}=1,112)$

\begin{tabular}{|c|c|c|c|c|c|}
\hline & $\begin{array}{c}\text { Underweight } \\
\left(<25^{\text {th }} \text { percentile }\right)\end{array}$ & $\begin{array}{c}\text { Low average-weight } \\
\left(25^{\text {th }} \geq \mathrm{BMI}<50^{\text {th }} \text { percentile }\right)\end{array}$ & $\begin{array}{c}\text { High average-weight } \\
\left(50^{\text {th }} \geq \mathrm{BMI}<85^{\text {th }} \text { percentile }\right)\end{array}$ & $\begin{array}{c}\text { Overweight } \\
\left(\mathrm{BMI} \geq 85^{\text {th }} \text { percentile) }\right.\end{array}$ & $p$ \\
\hline Girls & $32.7 \%$ & $27.5 \%$ & $31.3 \%$ & $8.5 \%$ & .027 \\
\hline Boys & $27.7 \%$ & $25.1 \%$ & $34.0 \%$ & $13.2 \%$ & \\
\hline Grade 7 & $33.6 \%$ & $27.2 \%$ & $27.2 \%$ & $12.0 \%$ & n.s. \\
\hline Grade 8 & $29.5 \%$ & $27.0 \%$ & $32.9 \%$ & $10.6 \%$ & \\
\hline Grade 9 & $27.6 \%$ & $24.7 \%$ & $38.8 \%$ & $9.0 \%$ & \\
\hline
\end{tabular}

Note. The BMI ranking was assigned using the WHO norms and weight status was classified following Jones \& Crawford (2006). 
Table 3 Main effects in appearance-related social pressure for gender

\begin{tabular}{ll}
$\begin{array}{l}\text { Girls } \\
(n=603)\end{array}$ & Boys \\
$(n=509)$ \\
$M$ & $M$ \\
$(S D)$ & $(S D)$ \\
\hline
\end{tabular}

\begin{tabular}{lll}
\hline Parental Pressure & & \\
\hline Parental Teasing & 1.18 & 1.11 \\
\cline { 2 - 3 } & $(0.48)$ & $(0.30)$ \\
\hline Injustice \& Ignorance & 1.13 & 1.14 \\
\cline { 2 - 3 } & $(0.33)$ & $(0.30)$ \\
\hline Parental Encouragement & 1.65 & 1.70 \\
\cline { 2 - 3 } & $(0.76)$ & $(0.74)$ \\
\hline Parental Norms \& Modeling & 2.12 & 2.12 \\
\hline & $(0.74)$ & $(0.76)$ \\
\hline
\end{tabular}

\begin{tabular}{|c|c|c|c|}
\hline \multicolumn{4}{|l|}{ Peer Pressure } \\
\hline \multirow[t]{2}{*}{ Peer Teasing } & 1.57 & 1.49 & $\eta^{2}=.01^{* * *}$ \\
\hline & $(0.68)$ & $(0.60)$ & \\
\hline \multirow[t]{2}{*}{ Exclusion } & 1.97 & 1.71 & $\eta^{2}=.05^{* * *}$ \\
\hline & $(0.82)$ & $(0.69)$ & \\
\hline \multirow[t]{2}{*}{ School \& Class Norms } & 2.18 & 1.99 & $\eta^{2}=.03 * * *$ \\
\hline & $(0.81)$ & $(0.71)$ & \\
\hline \multirow[t]{2}{*}{ Modeling by Friends } & 2.61 & 2.22 & $\eta^{2}=.06^{* * *}$ \\
\hline & $(0.76)$ & $(0.73)$ & \\
\hline
\end{tabular}

Note. The items are rated on a 5-point Likert scale from 1 (strongly disagree) to 5 (strongly agree).

${ }^{*} p<.05 ;{ }^{* *} p<.01 ;{ }^{* * *} p<.001$.

adolescents. In contrast to our hypothesis, differences emerged not only for modeling by friends, $F(2,1088)=$ $12.80, p<.01, \eta^{2}=.02$, and school and class norms, $F(2$, $1088)=35.29, p<.001, \eta^{2}=.06$, but also for peer teasing, $F(2,1088)=8.03, p<.001, \eta^{2}=.02$, and exclusion, $F(2,1088)=8.85, p<.001, \eta^{2}=.02$. Bonferroni post hoc tests were used to evaluate differences between gradelevels (corrected $p<.017$ ) and revealed that students from grade 7 reported significantly lower levels on all peer pressure scales compared to students from grades 8 or 9. Only regarding school and class norms could a significant difference be found between students from grades 8 and 9 . As reflected by the effect sizes, grade differences for school and class norms were particularly evident.

\section{Hypothesis 3}

Regarding variations by grade-level we expected that parental encouragement to control weight and shape would be more prevalent among older adolescents. The univariate follow-up analyses of grade differences (corrected $p<.006$ ) confirmed this hypothesis and a main effect for parental encouragement to control weight and shape revealed, which was perceived to a lesser degree in
Table 4 Main effects in appearance-related social pressure for grade

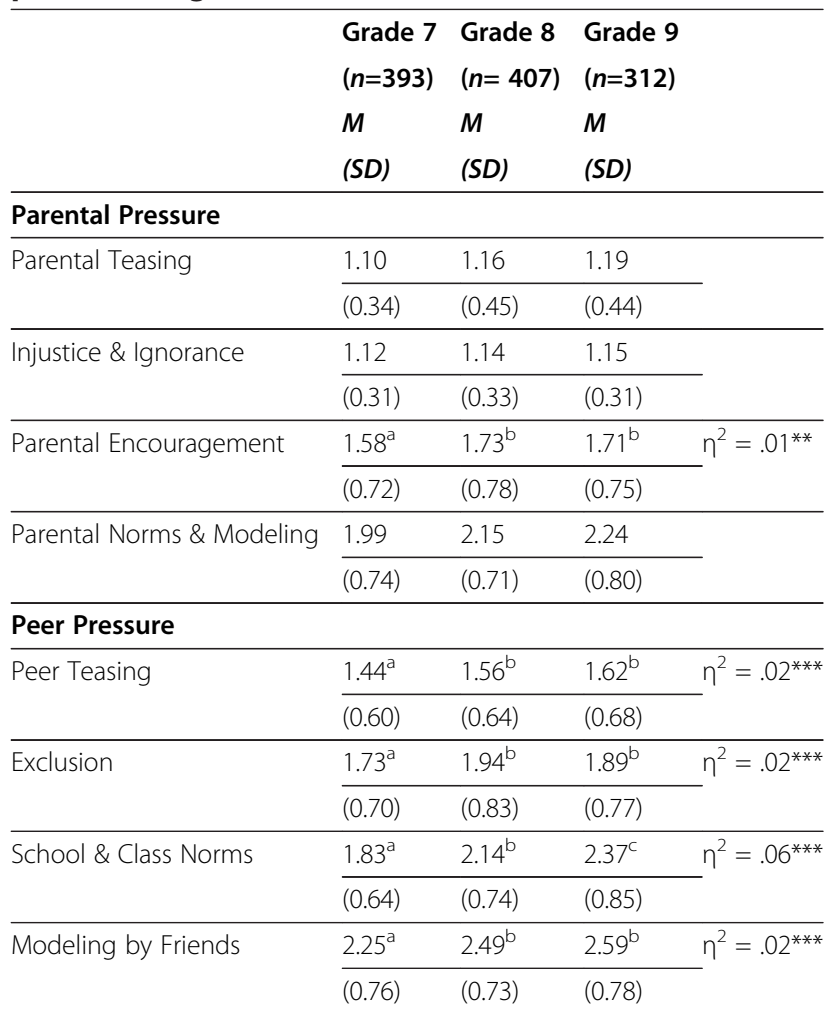

Note. The items are rated on a 5-point Likert scale from 1 (strongly disagree) to 5 (strongly agree). Means with the same subscript are not significantly different. ${ }^{*} p<.05 ;{ }^{* *} p<.01 ;{ }^{* * *} p<.001$

grade 7 compared to grade $8, F(2,1088)=6.48, p<.01$, $\eta^{2}=.01$.

\section{Main effects for body mass Hypothesis 4}

Finally, we predicted that overweight adolescents would report higher levels of all types of peer pressure. Univariate tests (corrected $p<.006$ ) combined with Bonferroni post hoc tests were used to evaluate differences between BMI categories (corrected $p<.008$ ). With regard to peer pressure, small effects for school and class norms, $F(3,1088)=5.56, p<.01, \eta^{2}=.02$, emerged. Interestingly, we found the highest scores among high-average-weight students. Post hoc tests indicated that high-average weight students scored significantly higher than underweight students on school and class norms. Main effects for peer teasing, $F(3,1088)=34.15, p<.001, \eta^{2}=.09$ and exclusion, $F(3$, $1088)=30.28, p<.001, \eta^{2}=.08$, proved to be particularly pronounced. Further, there emerged a trend, indicating that the level of peer teasing and exclusion increased with higher weight status. Underweight and low-average weight students displayed the lowest levels and did not differ in their scores. In contrast to our 
Table 5 Main effects in appearance-related social pressure for BMI - categories

\begin{tabular}{|c|c|c|c|c|c|}
\hline & $\begin{array}{l}\text { Under } \\
(n=338) \\
M \\
(S D)\end{array}$ & $\begin{array}{l}\text { Low } \\
(n=294) \\
M \\
(S D)\end{array}$ & $\begin{array}{l}\text { High } \\
(n=362) \\
M \\
(S D)\end{array}$ & $\begin{array}{l}\text { Over } \\
(n=118) \\
M \\
(S D)\end{array}$ & \\
\hline \multicolumn{6}{|l|}{ Parental Pressure } \\
\hline \multirow[t]{2}{*}{ Parental Teasing } & 1.11 & 1.13 & 1.18 & 1.22 & \\
\hline & $(0.34)$ & $(0.35)$ & $(0.45)$ & $(0.56)$ & \\
\hline \multirow{2}{*}{$\begin{array}{l}\text { Injustice \& } \\
\text { Ignorance }\end{array}$} & 1.09 & 1.13 & 1.18 & 1.15 & \\
\hline & $(0.22)$ & $(0.29)$ & $(0.39)$ & $(0.35)$ & \\
\hline \multirow{2}{*}{$\begin{array}{l}\text { Parental } \\
\text { Encouragement }\end{array}$} & $1.53^{\mathrm{a}}$ & $1.59^{\mathrm{a}}$ & $1.70^{\mathrm{a}}$ & $2.18^{\mathrm{b}}$ & $\eta^{2}=.07^{* * *}$ \\
\hline & $(0.66)$ & $(0.68)$ & $(0.77)$ & $(0.89)$ & \\
\hline \multirow{2}{*}{$\begin{array}{l}\text { Parental Norms \& } \\
\text { Modeling }\end{array}$} & 2.01 & 2.16 & 2.19 & 2.17 & \\
\hline & $(0.69)$ & $(0.73)$ & $(0.81)$ & $(0.78)$ & \\
\hline \multicolumn{6}{|l|}{ Peer Pressure } \\
\hline \multirow[t]{2}{*}{ Peer Teasing } & $1.38^{\mathrm{a}}$ & $1.43^{\mathrm{a}}$ & $1.62^{\mathrm{b}}$ & $1.98^{c}$ & $\eta^{2}=.09^{* * *}$ \\
\hline & $(0.49)$ & $(0.50)$ & $(0.68)$ & $(0.92)$ & \\
\hline \multirow[t]{2}{*}{ Exclusion } & $1.68^{\mathrm{a}}$ & $1.73^{\mathrm{a}}$ & $1.97^{b}$ & $2.29^{c}$ & $\eta^{2}=.08^{* * *}$ \\
\hline & $(0.62)$ & $(0.66)$ & $(0.85)$ & $(0.97)$ & \\
\hline \multirow{2}{*}{$\begin{array}{l}\text { School \& Class } \\
\text { Norms }\end{array}$} & $1.98^{\mathrm{a}}$ & $2.05^{\mathrm{ab}}$ & $2.22^{b}$ & $2.15^{\mathrm{ab}}$ & $\eta^{2}=.02^{* *}$ \\
\hline & $(0.70)$ & $(0.74)$ & $(0.83)$ & $(0.81)$ & \\
\hline \multirow{2}{*}{$\begin{array}{l}\text { Modeling by } \\
\text { Friends }\end{array}$} & 2.33 & 2.44 & 2.52 & 2.45 & \\
\hline & $(0.74)$ & $(0.76)$ & $(0.78)$ & $(0.80)$ & \\
\hline
\end{tabular}

Note. Under $=$ underweight $\left(\mathrm{BMI}<25^{\text {th }}\right.$ percentile), Low = low average $\left(25^{\text {th }} \leq \mathrm{BMI}<50^{\text {th }}\right.$ percentile), High $=$ high average $\left(50^{\text {th }} \leq \mathrm{BMI}<85^{\text {th }}\right.$ percentile), Over $=$ overweight $\left(\mathrm{BMI} \geq 85^{\text {th }}\right.$ percentile). The items are rated on a 5-point Likert scale from 1 (strongly disagree) to 5 (strongly agree). Means with the same subscript are not significantly different.

${ }^{*} p<.05 ;{ }^{* *} p<.01 ;{ }^{* * *} p<.001$.

hypothesis the different weight groups did not differ in the perception of modeling by friends.

\section{Hypothesis 5}

We further expected that overweight adolescents would experience more parental teasing and encouragement to control weight and shape. Regarding parental pressure, the results supported weight-related differences only for one aspect of parental pressure - encouragement to control weight and shape, $F(3,1088)=25.98, p<.001$, $\eta^{2}=.07$ - indicating a large effect. The trend suggested that parental encouragement to control weight and shape increased with higher weight status. However, the scores only significantly differed for overweight students.

To sum up, our analyses revealed main effects for gender, grade-level, and weight status, but no interaction between these factors. With an effect size of $\eta^{2}=.11$, gender differences proved to be particularly pronounced. Girls scored higher on all peer pressure scales and showed slightly higher scores on parental teasing. Moderate main effects for grade-level revealed that students from grade 7 differed from students from grades 8 and 9 on the peer pressure scales. Likewise, students from grade 7 showed low levels of parental encouragement to control weight and shape. Finally, main effects for weight status were particularly pronounced for peer teasing and exclusion as well as for parental encouragement to control weight and shape. The findings indicated that particularly high-average and overweight adolescents perceived appearance pressure.

\section{Discussion}

The relevance of appearance-related social pressure as a crucial factor for low self-esteem and depression as well as body dissatisfaction and unhealthy body change efforts has been proven repeatedly [e.g.44-46]. Up to now, knowledge of gender, weight, and age-related variations in social pressure has either been incomplete or controversial because very few studies have explicitly investigated these aspects together. Moreover, most of the existing studies have permitted only limited conclusions, because they either focused on single aspects of social pressure or were limited in their assessment.

The current study thus contributes to a better understanding of the occurrence of social pressure by explicitly addressing gender, grade-level, and weight variations in a large sample of German adolescent girls and boys. Furthermore, we applied a new measure (FASD), whose psychometric quality and applicability for both girls and boys has been proven before $[17,43]$ and which allows a broad assessment of aspects of appearance pressure from both peers and parents. In doing so, the results may help to identify adolescents who are particularly at risk of suffering from appearance-related social pressure and thus provide concrete advice for preventive approaches.

Following the overall effects of the current study, the findings suggest that social pressure is more prevalent during mid-adolescence compared to early adolescence and girls and adolescents with higher weight are particularly affected. A comparison of the effect sizes indicated that gender differences were particularly pronounced in the current sample.

\section{Gender variations}

Our hypotheses regarding gender differences in peer and parental pressure were only partly supported. While we found the expected gender differences on all peer pressure scales, gender effects were only found for parental teasing. Thus, our results support previous findings on negative verbal commentary that found a higher prevalence among girls $[6,14]$. Nevertheless, the conclusion often drawn in previous research that the parental impact is generally higher for girls was probably premature. Because the effect size for parental teasing was rather low and no effects emerged on the other scales, levels of parental pressure among girls and boys seem to be more 
similar than previously assumed. This finding also resembles the results of Rodgers et al. [24]. Even though they found gender differences for a few aspects of parental pressure, a closer look at the scores and effect sizes reveals that only the difference regarding negative maternal comments is noteworthy. Maybe no effects were revealed because of the extreme floor effect and the restricted variance of these FASD scales, which is also known for instruments assessing similar constructs in population-based samples [6].

Gender effects for peer pressure are in line with current research, indicating that girls are more strongly affected by peer influences and the impact of friends is especially important $[7,46]$. Gender effects with regard to teasing experiences have been controversial because of limitations in the measurement of teasing. Our results obtained with a gender-neutral, reliable peer teasing scale support the findings of the American EAT-Project [26] and can serve as further evidence that girls experience more peer teasing. Summing up, the results support the assumption that girls are particularly embedded in an appearance culture $[1,46]$. In detail, the findings suggest that girls perceive more pressure from appearance norms and modeling and are more often subject to proximate forms of peer pressure such as teasing or exclusion. Because the current study applied a measure of social pressure that is not biased by female ideals and has proven to be suitable for both girls and boys alike, we conclude that the higher extent of appearance pressure among females is not just a result of inappropriate measurement but in fact a result of the greater societal emphasis on beauty and appearance for females [5].

\section{Grade-level variations}

The prevalence of appearance-related social pressure especially by peers underlies age-related trends whereas grade-level effects in parental pressure only emerged for encouragement to control weight and shape and were also rather minor.

In contrast to Chen and Jackson [31], these effects proved to be comparable for girls and boys in this German sample. Based on previous results, comparing male body image in Western and Asian cultures, we assume that the divergent results might point to a cultural difference. As Yang, Grey, and Pope [47] revealed, Asian males were less preoccupied with body image than Western males and they discussed interesting reasons why in Western cultures more emphasis is placed on male appearance (e.g. media exposure, decline in traditional male roles).

In accordance with the literature [7,32], differences in our sample were particularly evident comparing early (grade 7) and middle adolescents (grades 8 and 9). Although conclusions must be drawn cautiously due to the cross-sectional design, it seems as if the transition from grade 7 to grade 8 is particularly relevant. Interestingly, differences were mainly reflected by an increase of perceived school and class norms. This effect might be a result of the local school system. In this region of Germany, students change schools between grades 6 and 7. Hence, the adolescents in grade 7 have just joined a new school context and their new schoolmates. This new school context constitutes an important developmental transition, which is associated with changes in social roles and a substantial reorganization of attitudes and beliefs and has been considered a period of risk for problematic behavior [48]. Thus, we believe that among grade 7 students the establishment of norms and group processes has presumably just started. Consequently, we assume that the results probably reflect both - on the one hand, individual changes and transitions throughout adolescence, and on the other hand, the development of the class as a group of shared attitudes and values. It might be an interesting issue for future studies to distinguish between these two processes and figure out which role age per se or the attainment of a certain grade-level plays in this issue. Furthermore, the findings suggest that early adolescence as well as school transitions are crucial periods for establishing prevention programs that counteracts the development of an appearance culture within a class. Again, we have to emphasize that the findings can only lead to cautious conclusions because of the cross-sectional design of the study. Longitudinal studies are needed to confirm these findings.

\section{Body mass variations}

Our results suggest that mainly high-average and overweight adolescents experience more appearance pressure from peers and parents, whereas teasing and exclusion are particularly prevalent. We could not replicate the interaction of weight and gender reported by Jones and Crawford [7], who hypothesized that girls experience teasing for higher weight whereas boys are teased for being underweight. In line with stigmatization research [49] and our expectations, the results suggest that overweight adolescents are generally faced with more appearance pressure regardless of their gender. A possible explanation for this is methodological, for we could also find slight similar tendencies in the univariate but not in the multivariate analysis. Jones and Crawford [7] also used univariate analyses. However, due to the intercorrelations between the aspects of pressure, we decided to use a multivariate and thus, more conservative approach, which reveals that the interactive effects are not strong enough and that only the main effect of BMI is relevant. Thus, our findings indicate that girls and boys with higher weight are equally at risk of being faced with appearance pressure.

Body mass variations in the perception of more subtle, norm-related aspects of pressure have rarely been 
investigated and could only be observed to a lesser extent in our sample. However, small effects for school and class norms indicated that high-average students show the highest levels. Possibly, adolescents who barely fail to fit the slim norm are more likely to internalize appearance ideals [46] and are thus more sensible to subtle appearance-related messages.

Regarding parental pressure the body mass effect is primarily reflected in higher levels of parental encouragement to control weight and shape especially among overweight participants. Hence, overweight adolescents perceive their parents as more demanding regarding weight or shape control. This result is not surprising, because parents are often concerned about the overweight of their child and feel responsible [50]. So, they probably try to support weight control and dieting efforts with comments designed to act as reminders. In accordance with previous studies $[13,15,25]$ our findings can serve as further evidence that these encouraging messages are more problematic than previously assumed. The results indicate that the line is fine between support and pressure and future research must keep track of possible consequences. Beyond this, the findings appear to be particularly relevant for the field of obesity prevention and treatment of children and adolescents. Approaches including parents should address these processes and negotiate the balancing act in teaching parents to support their children without putting them under pressure.

The results of our study are limited to a certain extent first due to the sample. Unfortunately, we could not use the data collected on SES and ethnicity of the sample, because plausibility checks revealed that some adolescents misunderstood these items. Hence, we had to consult data from the Federal Statistical Office, which show that Potsdam is a city with a low percentage of inhabitants with foreign backgrounds and a high percentage of inhabitants with academic and higher social background. Because we only included students from schools with higher educational levels generalizations are restricted and future research might extend these findings to larger, more representative samples. Furthermore, the use of self-reported weight remains a limitation when investigating body mass variations. However, self-reported weight has repeatedly proven to be a valid measure in epidemiological studies with adolescents [12,36]. In addition, BMI confounds lean mass with fat mass, which might lead to a screwed picture when studying males. Therefore future research should also include fat-free mass, body fat indices or girth measurements in order to confirm these findings. Finally, the results are based on cross-sectional data and thus do not permit developmental conclusions. The age-related variations can only point to possible trends that require further confirmation in longitudinal studies.

\section{Conclusion}

By investigating a broad range of aspects of social pressure in a large sample of adolescent girls and boys, the current study points to interesting issues regarding age-, gender-, and weight-based risks for appearance-related social pressure. On this basis the results of the current study could enhance the current state of theory on appearance-related social pressure and pointed out the following:

a) Girls in general are not more affected by social pressure. Differences in parental pressure seem negligible. However, gender variations regarding peer pressure are noteworthy.

b) Older students experience more peer pressure. The crucial moment seems to be the transition from grade 7 to grade 8 . Age-related variation in parental pressure did not notably occur.

c) Higher weight is associated with higher levels of proximate individual-related appearance pressure (e.g. teasing, exclusion, and parental encouragement), while effects regarding norm-related forms of pressure were rather small. An interaction of weight and gender could not be replicated.

The findings provide suggestions for preventive efforts. Approaches are needed that strengthen those adolescents who are particularly at risk - in our study, these were girls and adolescents with higher weight status. At the same time the results point to the relevance of peers in the exertion of appearance pressure. Hence preventive approaches should bring up the topic of appearance pressure in a school-based context, since early adolescence and school transition appear to be crucial periods for these efforts.

\section{Competing interests}

The authors declare they have no competing interests.

\section{Authors' contribution}

SH conceived the study, participated in the design and data collection, performed the statistical analyses and drafted the manuscript. PW designed the project in which the study was conducted, obtained funding, participated in its design and coordination and supervised the data analyses and the writing process. All authors read and approved the final manuscript.

\section{Acknowledgements}

This study is supported by the German Federal Ministry of Education and Research (BMBF, Registr.nr.: 01EL0607) and the University of Potsdam, Germany. We are grateful to the students, parents and teachers who agreed to participate in the study. Special thanks to Eva-Maria Krentz, Sebastian Mohnke, Katja Kröller and all the other members of our research team for their support at the different stages of the study.

Received: 27 November 2012 Accepted: 2 May 2013

Published: 17 May 2013

\section{References}

1. Jones DC: Body image among adolescent girls and boys: A longitudinal study. Dev Psychol 2004, 40:823-835. 
2. Ricciardelli LA, McCabe MP: Self-esteem and negative affect as moderators of sociocultural influences on body dissatisfaction, strategies to decrease weight, and strategies to increase muscles. Sex Roles 2001 44:189-207.

3. Smolak L: Body image in children and adolescent: Where do we go from here? Body Image 2004, 1:15-28.

4. Thompson JK, Heinberg LJ, Altabe M, Tantleff-Dunn S: Exacting beauty: Theory, assessment, and treatment of body image disturbance. Washington, DC: American Psychological Association; 1999.

5. Stice E: Review of the evidence for a sociocultural model of bulimia nervosa and an exploration of the mechanisms of action. Clin Psychol Rev 1994, 14:633-661.

6. Phares V, Steinberg A, Thompson J: Gender differences in peer and parental influences: Body image disturbance, self-worth, and psychological functioning in preadolescent children. $J$ Youth Adolesc 2004, 33:421-429.

7. Jones DC, Crawford J: The peer appearance culture during adolescence: Gender and body mass variations. J Youth Adolesc 2006, 35:257-269.

8. Mackey ER, La Greca AM: Does this make me look fat? Peer crowd and peer contributions to adolescent girls' weight control behaviors. J Youth Adolesc 2008, 37:1097-1110.

9. Hayden-Wade HA, Stein RI, Ghaderi A, Saelens BE, Zabinski MF, Wiffley DE: Prevalence, characteristics, and correlates of teasing experiences among overweight children vs. non-overweight peers. Obes Res 2005, 13:1381-1392.

10. Rodgers RF, Chabrol H: Parental attitudes, body image disturbance and disordered eating amongst adolescents and young adults: A review. Eur Eat Disord Rev 2009, 17:137-151.

11. Agras WS, Bryson S, Hammer LD, Kraemer HC: Childhood risk factors for thin body preoccupation and social pressure to be thin. J Am Acad Child Adolesc Psychiatry 2007, 46:171-178.

12. Field AE, Camargo CA, Taylor CB, Berkey CS, Roberts SB, Colditz GA: Peer, parent, and media influences on the development of weight concerns and frequent dieting among preadolescent and adolescent girls and boys. J Pediatr 2001, 107:54-60.

13. Meesters C, Muris P, Hofnagel C, van Gemert M: Social and family correlates of eating problems and muscle preoccupation in young adolescents. Eat Behav 2007, 8(1):83-90.

14. Smolak L, Levine MP, Schermer F: Parental input and weight concerns among elementary school children. Int J Eat Disord 1999, 25:263-271.

15. Kluck AS: Family influence on disordered eating: The role of body image dissatisfaction. Body Image 2010, 7:8-14.

16. Vincent M, McCabe MP: Gender differences among adolescents in family, and peer influences on body dissatisfaction, weight loss, and binge eating behaviors. J Youth Adolesc 2000, 29:205-221.

17. Helfert $S$, Warschburger P: Fragebogen zum aussehensbezogenen sozialen Druck (FASD). Klinische Diagnostik und Evaluation 2009, 3:207-221.

18. Lawler M, Nixon E: Body dissatisfaction among adolescent boys and girls: The effects of body mass, peer appearance culture and internalization of appearance ideals. J Youth Adolesc 2010, 40(1):59-71.

19. Oliver KK, Thelen MH: Children's perceptions of peer influence on eating concerns. Behav Ther 1996, 27:25-39.

20. Crosnoe R, Frank K, Strassmann A: Gender, body size and social relations in american high schools. Soc Forces 2008, 86:1189-1216.

21. McCreary DR, Sasse DK: An exploration of the drive for muscularity in adolescent boys and girls. J Amer Coll Health 2000, 48:297-305.

22. Ricciardelli LA, McCabe MP: Pursuit of muscularity among adolescents. In The muscular ideal. Psychological, social and medical perspectives. Edited by Thompson JK, Cafri G. Washington D.C: American Psychological Association; 2007:199-216.

23. Ricciardelli LA, McCabe MP: Sociocultural and individual influences on muscle gain and weight loss strategies among adolescent boys and girls. Psychol Schools 2003, 40:209-224.

24. Rodgers RF, Faure K, Chabrol H: Gender differences in parental influences on adolescent body dissatisfaction and disordered eating. Sex Roles 2009, 61:837-849

25. Wertheim EH, Martin G, Prior M, Sanson A, Smart D: Parent influences in the transmission of eating and weight related values and behaviors. Eat Disord J Treat Prev 2002, 10:321-334.

26. Neumark-Sztainer D, Falkner N, Story M, Perry C, Hannan PJ, Mulert S: Weight-teasing among adolescents: Correlations with weight status and disordered eating behaviors. Int J Obes 2002, 26:123-131.
27. Ata RN, Ludden AB, Lally MM: The effects of gender and family, friend, and media influences on eating behaviors and body image during adolescence. J Youth Adolesc 2007, 36:1024-1037.

28. Fend H: Eltern und Freunde: Soziale Entwicklung im Jugendalter. Bern: Huber 1998.

29. Hill JP, Lynch ME: The intensification of gender-related role expectations during early adolescence. In Girls at puberty: Biological and psychological perspectives. Edited by Brooks-Gunn J, Petersen A. New York: Plenum; 1983:201-228.

30. Dohnt HK, Tiggemann M: Body image concerns in young girls: The role of peers and media prior to adolescence. J Youth Adolesc 2006, 35(2):141-151.

31. Chen J, Jackson T: Gender and age group differences in mass media and interpersonal influences on body dissatisfaction among Chinese adolescents. Sex Roles 2012, 66:3-20.

32. Andersson B: Development trends in reaction to social pressure form adults versus peers. Int J Behav Dev 1979, 2:269-286.

33. Bulcroft RA: The value of physical change in adolescence: Consequences for the parent-adolescent exchange relationship. J Youth Adolesc 1991, 20(1):89-105.

34. Striegel-Moore R, Kearney-Cooke A: Exploring parents' attitudes and behaviors about their children's physical appearance. Int J Eat Disord 1994, 15:377-385

35. Berger U, Schilke C, Strauß B: Gewichtssorgen und Diätverhalten bei Kindern in der 3. und 4. Klasse. Psychother Psych Med 2005, 7:331-338.

36. Fonseca H, Silva AM, Matos MG, Esteves I, Costa P, Guerra A, Gomes-Pedro J: Validity of BMI based on self-reported weight and height in adolescents. Acta Paediatr 2010, 99:83-88.

37. WHO: Growth references 5-19 years. 2010. http://www.who.int/growthref/ who2007_bmi_for_age/en/.

38. Stice $E$, Bearman SK: Body image and eating disturbances prospectively predict growth in depressive symptoms in adolescent girls: A growth curve analysis. Dev Psychol 2001, 37:597-607.

39. Young EA, Clopton JR, Bleckley MK: Perfectionism, low self-esteem, and family factors as predictors of bulimic behavior. Eat Behav 2004, 5:273-283.

40. Thompson JK, Cattarin J, Fowler B, Fisher E: The Perception of Teasing Scale (POTS): A revision and extension of the Physical Appearance Related Teasing Scale (PARTS). J Pers Assess 1995, 65:146-157.

41. McCabe MP, Ricciardelli LA: Sociocultural influences on body image and body changes among adolescent boys and girls. J Soc Psych 2000, 143:5-26.

42. Presnell K, Bearman SK, Stice E: Risk factors for body dissatisfaction in adolescent boys and girls: A prospective study. Int J Eat Disord 2004, 36:389-401.

43. Helfert S, Warschburger P: A prospective study on the impact of peer and parental pressure on body dissatisfaction in adolescent girls and boys. Body Image 2011, 8(2):101-109.

44. Wichstrom $L$ : The emergence of gender differences in depressed mood during adolescence: The role of intensified gender socialization. Dev Psych 1999, 35:232-245.

45. Wade TD, Lowes J: Variables associated with disturbed eating habits and overva- lued ideas about the personal implications of body shape and weight in a female adolescent population. Int J Eat Disord 2002, 32:39-45.

46. Jones DC, Vigfusdottir T, Lee Y: Body image and the appearance culture among adolescent girls and boys: An examination of friend conversations, peer criticism, appearance magazines, and the internalization of appearance ideals. J Adolesc Res 2004, 19:323-339.

47. Yang CF, Grey P, Pope HG: Male body image in Taiwan versus the West: Yanggang Zhiqi meets the Adonis complex. Am J Psychiartry 2005, 162:263-269.

48. Smolak L, Levine MP: Adolescent transitions and the development of eating disorders. In The developmental psychopathology of eating disorders: Implications for research, prevention and treatment. Edited by Smolak L, Levine ML, StriegelMoore R. Mahwah, NJ: Lawrence Erlbaum Associates; 1996:207-234.

49. Kraig KA, Keel PK: Weight-based stigmatization in children. Int J Obes 2001, 25:1661-1666.

50. Jackson D, Wilkes L, McDonald G: If I was in my daughter's body I'd be feeling devastated: Women's experiences of mothering an overweight or obese child. J Child Health Care 2007, 11:29-39.

doi:10.1186/1753-2000-7-16

Cite this article as: Helfert and Warschburger: The face of appearancerelated social pressure: gender, age and body mass variations in peer and parental pressure during adolescence. Child and Adolescent Psychiatry and Mental Health 2013 7:16. 